\title{
ON THE LOGARITHMIC DERIVATIVES
}

\author{
OF SUBORDINATE FUNCTIONS
}

\author{
M.M. ELHOSH
}

Coefficient and integral mean bounds are obtained for functions subordinate to close-to-convex functions.

Let $C$ denote the class of close-to-convex functions $f(z)$ normalised so that $f(0)=f^{\prime}(0)-1=0$ and satisfying $\operatorname{Re} z f^{\prime}(z) / e^{i \alpha_{\phi}}(z) \geq 0(|\alpha| \leq \pi / 2)$ for some starlike function $\phi(z)$ normalized as above and satisfying $\operatorname{Re} z \phi^{\prime}(z) / \phi(z) \geq 0$ in $|z|<1$, $\left[6\right.$, p.17]. We denote the class of all $\phi(z)$ by $S^{*}$. It is well known that $S^{*}$ is contained in $C$ [6, pp.11-18]. Now let $S(C)=\{f: f<g$ for some $g$ in $C$ \} where $f<g$ means $f$ is subordinage to $g$. Such a relation is usually written in the form $f(z)=g(w(z))$, where $w(0)=0$ and $|w(z)|<1$ in $|z|<1$.

We define $S\left(S^{*}\right)$ similarly; clearly $S\left(S^{*}\right) \subseteq S(C) \quad[6, p .72]$. We now apply the *-function and the extreme point theory arguments due to Baemstein [1] (see also [2], [4], [5], [8]) and MacGregor [9] (see also [6, Chapter 6], [5]) respectively to prove the following results.

Received 19 August, 1987.

Copyright Clearance Centre, Inc. Serial-fee code: 0004-9727/87 $\$ A 2.00+0.00$. 
THEOREM 1. Let $f \in S(C)$ and $p$ be a positive integer; then for $z=r e^{i \theta}, 0<r<1$, we have $\int_{0}^{2 \pi}\left|z f^{\prime}(z) / f(z)\right|^{2 p} d \theta \leq \int_{0}^{2 \pi}|(1+2 z) /(1-z)|^{2 p} d \theta=0\left((1-r)^{1-2 p}\right), \quad(r \rightarrow 1)$. Also for $-\infty<p<\infty$ we have

$$
\int_{0}^{2 \pi}\left|f^{\prime}(z)\right|^{p} d \theta \leq \int_{0}^{2 \pi}\left|K^{\prime}(z)\right|^{p} d \theta
$$

where $K(z)$ is the Koebe function.

Proof of Theorem 1. We see from [6, Chapter 6] (see in particular Theorems 6.11 and 6.12 ) that it suffices to prove the theorem for functions of the form

$$
f(z)=w\left[z-\frac{1}{2}(x+y) z^{2}\right] /(1-y z)^{2},
$$

where $|w|=|x|=|y|=1$ and $x \neq y$.

Indeed we see from this that $\left|z f^{\prime}(z) / f(z)\right| \leq 3 /(1-r)$ which implies that the family $\left\{z f^{\prime}(z) / f(z): f \in S(C)\right\}$ is compact and hence, as in [9, pp. 361-365], has compact closed convex hull and consequently the extreme points of the closed convex hull of this family are contained in it.

Thus we deduce from the logarithmic derivative of (1) that

$$
\begin{aligned}
\frac{z f^{\prime}(z)}{f(z)} & =\frac{1+y z}{1-y z}-\frac{(x+y) z}{2-(x+y) z} \\
& <(1+2 z) /(1-z)
\end{aligned}
$$

where, as in $[7, \mathrm{p} .52], \ll$ means that if $\sum a_{n} z^{n} \ll \Sigma C_{n} z^{n}$ then $\left|a_{n}\right| \leq c_{n}, n=0,1,2, \ldots$.

Using this and [7, Lemma 2.4.1] we deduce that if $p$ is a positive integer then $\left[z f^{\prime}(z) / f(z)\right]^{p} \ll[(1+2 z) /(1-z)]^{p}$ which means that if 
$\left[z f^{\prime}(z) / f(z)\right]^{p}=\Sigma C_{n}(p) z^{n}$ and $[(1+2 z) /(1-z)]^{p}=\Sigma D_{n}(p) z^{n}$ then $\left|C_{n}(p)\right| \leq D_{n}(p), n=0,1,2, \ldots$.

Thus we see from Parseval's identity that

$$
\begin{aligned}
\int_{0}^{2 \pi}\left|z f^{\prime}(z) / f(z)\right|^{2 p} d \theta & =2 \pi \sum_{n=0}^{\infty}\left|c_{n}(p)\right|^{2} r^{2 n} \\
& \leq 2 \pi \sum_{n=0}^{\infty} D_{n}^{2}(p) r^{2 n}=\int_{0}^{2 \pi}|(1+2 z) /(1-z)|^{2 p} d \theta .
\end{aligned}
$$

This gives the first inequality of Theorem 1 .

To prove the second inequality we apply the *-function argument as in the proof of Theorem I of [8], (see also [1], [2], [4, Chapter 7 ) to the derivative of $(1), f^{\prime}(z)=w(1-x z) /(1-y z)^{3}$. We deduce (in this connection see the note about the rearrangement on page 84 of [6]) that

$$
\begin{aligned}
\left( \pm \log \left|f^{\prime}(z)\right|\right) * & =\left( \pm \log \left|w(1-x z) /(1-y z)^{3}\right|\right) * \\
& \leq( \pm \log |w(1-x z)|) *+3( \pm \log |1 /(1-y z)|) * \\
& =( \pm \log |1 \pm z|) *+3( \pm \log |1 /(1 \mp z)|) * \\
& =\left( \pm \log \left|K^{\prime}(z)\right|\right) * .
\end{aligned}
$$

The second inequality of Theorem 1 now follows by applying [1, Proposition 3] with $\phi(u)=\exp [p u]$ just as in [1, Theorem 1], [2, Theorem 1], [4, Theorem 7.2], [5] and [8].

THEOREM 2. Let $f \in S\left(S^{*}\right)$; then for $z=r e^{i \theta}, 0<r<1$, and $-\infty<p<\infty$ we have

$$
\int_{0}^{2 \pi}\left|z f^{\prime}(z) / f(z)\right|^{p} d \theta \leq \int_{0}^{2 \pi}\left|z K^{\prime}(z) / K(z)\right|^{p} d \theta
$$

The proof of Theorem 2 follows by applying the same method used in the proof of the second inequality of Theorem 1 to the function $f(z)=x z /(1-y z)^{2}$ where $|x|=|y|=1[6$, Theorem 5.22]. 
THEOREM 3. Let $f \in S(C)$ and $f(z)=z+\sum_{n=2}^{\infty} a_{n} z^{n}$; then for $n \geq 1$ we have

$$
\begin{aligned}
& || a_{n}|-| a_{n-1}|| \leq 1, \\
& |n| a_{n}|-(n-1)| a_{n-1}|| \leq 2 n-1, \\
& \left|a_{n} a_{n+2}-a_{n+1}^{2}\right| \leq 5 .
\end{aligned}
$$

Proof of Theorem 3. We see from [6, p. 72] that we may consider functions of the form ( 1 ) which have coefficients

$$
a_{n}=\frac{2}{2} w[(n+1) y-(n-1) x] y^{n-2}
$$

where as in (1) $|w|=|x|=|y|=1$ and $x \neq y$.

From (2) we immediately see that

$$
\left|a_{n}-y a_{n-1}\right|=\frac{1}{2}|x-y| \leq 1
$$

The first inequality of Theorem 3 now follows from (3) since ||$a_{n}|-| a_{n-1}|| \leq\left|a_{n}-y a_{n-1}\right|$ when $|y|=1$. Similarly, we prove the second inequality by using (2). To prove the second inequality we differentiate (1) and deduce that

$$
\begin{aligned}
(1-y z)^{2} f^{\prime}(z) & =w(1-x z) /(1-y z) \\
& \ll(1+z) /(1-z)
\end{aligned}
$$

This implies, in view of the definition of $\ll$ above, that

$$
\left|n a_{n}-2(n-1) y a_{n-1}+(n-2) y^{2} a_{n-2}\right| \leq 2
$$

From (3) and (4) we deduce for some fixed $y(|y|=1)$ satisfying (1), (2), (3) and (4) that

$$
\begin{aligned}
\left|a_{n} a_{n+2}-a_{n+1}^{2}\right|=\mid \frac{a_{n}}{n}\left[(n+2) a_{n+2}-2(n+1) y a_{n+1}+n y^{2} a_{n}\right] & -\left(a_{n}-y a_{n-1}\right)^{2} \\
& -\frac{2 a_{n}}{n}\left(a_{n+2}-y a_{n+1}\right) \mid
\end{aligned}
$$




$$
\leq 5 \text {, }
$$

where we have used the inequality $\left|a_{n}\right| \leq n$ which follows from (2). This completes the proof of Theorem 3 .

\section{Remarks}

1. We note that the first inequality of Theorem 3 is known where $f$ belongs to $S^{*}[3, \mathrm{p} .537]$ while the second and third inequalities are known where $f$ belongs to $C[3, \mathrm{p} .537]$ and $[10]$.

2. The second inequality of Theorem 1 extends Theorem 6.12 of [6] for $f^{\prime}$. However if $f(z)=g(z)$ in Theorem 1 , then the second inequality is due to Leung [8] and the first is due to the author [5] when $p=2$.

3. The Koebe function and its rotations show that the second inequality of Theorem 1 and Theorem 2 as well as the first two inequalities of Theorem 3 are sharp. The first inequality of Theorem $I$ is not sharp and it is quite likely that Theorem 2 holds for the wider class $S(C)$. It is also possible that $\left|a_{n} a_{n+2}-a_{n+1}^{2}\right| \leq 1$ in Theorem 3. This is certainly true for $S\left(S^{*}\right)$ as can be seen from the representation $f(z)=x z /(1-y z)^{2}$ of Theorem 2 .

\section{References}

[1] A. Baernstein, "Integral means, univalent functions and circular syrmetrization", Acta Math. 133 (1974), 129-162.

[2] A. Baernstein and J. Brown, "Integral means of derivatives of monotone slit mappings", Comment. Math. Helv. 57 (1982), 331-348.

[3] D. Brannan and J. Clunie, Aspects of Contemporary Complex Analysis, (Academic Press, 1980).

[4] P. Duren, Univalent Functions, (Springer-Verlag, New York, 1983).

[5] M.M. Elhosh, "On the logarithmic derivatives of close-to-convex functions", Math. Jopon. (to appear)

[6] D. Hallenbeck and T. MacGregor, Linear Problems and Convexity Techniques in Geometric Function Theory, (Pitman Publ. Ltd., 1984l. 
[7] E. Hille, Ordinary differential equations in the complex domain, (John Wiley, 1976).

[8] Y. Leung, "Integral means of derivatives of some univalent functions", Bull. London Math. Soc. 11 (1979, 289-284.

[9] T. MacGregor, "Applications of extreme-point theory to univalent functions", Michingan Math. J. 19 (1972), 361-376.

[10] K. Noor, "On the Hankel determinants of close-to-convex univalent functions", Internat. J. Math. Math. Sci. 3 (1980), 477-481.

Pure Mathematics Department,

The University College of Wales,

Aberys twyth

Dyfed

United Kingdom. 